\title{
Effect of iron supplementation on intestinal function and oxidative stress in piglets with induced colitis ${ }^{*}$
}

\author{
Q. Chen ${ }^{1,2}$, G.W. Le ${ }^{1,3}$, Y.H. Shi ${ }^{1}$, Sh.M. Zhang ${ }^{2}$ and X. Jin ${ }^{2}$ \\ ${ }^{1}$ Key Laboratory of Food Science and Safety, Ministry of Education, Southern Yangtze University \\ Wuxi 214036, P.R. China \\ ${ }^{2}$ Institute of Animal Science, Jilin Academy of Agricultural Science \\ Gongzhuling 136100, P.R. China
}

(Received 19 June 2006; revised version 24 October 2006; accepted 2 May 2007)

\begin{abstract}
To estimate the effect of oral iron on the intestinal function and oxidative redox status in piglets with dextran sulphate sodium (DSS)-induced colitis, fifty 21-day-old weaned piglets were randomly divided into five groups of ten each. The piglets received regular diet and water, or four diets with $0,0.03,0.2$ or $1 \% \mathrm{FeSO}_{4} \cdot 2 \mathrm{H}_{2} \mathrm{O}$ and water with $4 \%$ DSS, respectively, for 10 days. We observed a markedly higher lactulose/mannitol (Lac/Man) excretion ratio and lower serum concentration of D-xylose in Fe 0.2 and 1\% supplementated piglets than piglets on DSS alone, but not in Fe $0.03 \%$ supplemented piglets. The activities of superoxide dismutase (SOD), catalase (CAT) and glutathione peroxidase (GPx) significantly increased in the DSS group compared with the control group. Iron supplementation significantly decreased the activities of GPx and CAT, as was vitamin E content, but not SOD. Furthermore, excess iron significantly increased the production of malondialdehyde (MDA) and hydroperoxides (HP), in a dose-response related manner.
\end{abstract}

KEY WORDS: piglet, $\mathrm{FeSO}_{4}$, colon, permeability, antioxidation enzyme

\section{INTRODUCTION}

Early weaned piglets are faced with the serious problem of inflammatory bowel disease (IBD) in modern pig production, and IBD usually leads to diarrhoea and iron-deficiency anaemia. Iron is an essential nutrient and its sufficient intake is

\footnotetext{
* Supported by the National Natural Science Foundation of China, Grant No. 30571347

${ }^{3}$ Corresponding author: e-mail: legw16@yahoo.com.cn
} 
necessary for normal functioning of many processes in the body. Its deficiency is associated with impaired functioning of the immune system (Bowlus, 2003). Iron deficiency apparently negatively influenced the immunocompetence in piglets (Svoboda et al., 2004). Nutritional or iron-deficiency anaemia has long been recognized as a serious problem in pig production. Piglets with iron-deficiency anaemia are prone to diarrhoea, often associated with Escherichia coli infections (Daykin et al., 1982). Accordingly it is necessary for piglets with IBD to add iron to the diet. However, excess iron can be lethal because it facilitates free radical formation. These may be involved in enhanced oxidative stress due to HaberWeiss reaction (Imlay et al., 1988). Hydrogen peroxide $\left(\mathrm{H}_{2} \mathrm{O}_{2}\right)$ and superoxide $\left(\mathrm{O}_{2}^{-}\right)$were produced by activated neutrophils in the gastrointestinal mucosa with IBD (Grisham and Granger, 1988). $\mathrm{H}_{2} \mathrm{O}_{2}$ then reacts further in the presence of catalytic amounts of trace iron to produce the highly reactive and damaging species, hydroxyl radical $(\mathrm{OH} \cdot)$, by the Haber-Weiss reaction. $\mathrm{OH} \cdot$ is considered to be the most reactive species in the reactive oxygen species (ROS). It is highly reactive and has the ability to modify intracellular processes. The acute stress and chronic stress can induce gastrointestinal oxidative stress and mucosal injury through enhanced production of ROS (Manashi et al., 2000). Oxidative damage amplifies intestinal inflammation and results in a virulent cycle of oxidative stress and inflammation. Excessive iron supplementation has been demonstrated to produce disease exacerbation (Erichsen et al., 1998). Srigiridhar et al. (2001) have earlier shown that iron-deficient intestine is more susceptible to iron-mediated oxidative damage than normal intestine due to increased production of hydroxyl radicals.

The susceptibility of a given organ to oxidative stress depends on its antioxidant defence status, and an overall balance between oxidants and antioxidants is required to maintain cellular homeostasis. The body's antioxidant system includes naturally occurring small antioxidant molecules, such as ascorbic acid, $\alpha$-tocopherol, and antioxidant enzymes, such as SOD, CAT and GPx. However, after long episodes of colitis, sustained oxidative stress would weaken the capability of endogenous antioxidants. Oxidative injury is caused by a disturbance of the sophisticated oxidant-antioxidant balance and occurs when oxidants overwhelm the endogenous antioxidant defence mechanisms (Urban et al., 1995). The damaging effects of reactive oxygen species on cellular biomolecules are well documented and the consequences of such have been implicated in the etiology of a number of organism disorders (Brennan et al., 2000).

As pigs are farm animals, it is necessary to know more precisely how excessive iron influences the intestinal function of the piglets, especially the piglets with IBD. In the present study, using a piglet model with DSS-induced colitis, we examined the effect of iron supplementation on intestinal function and oxidative stress parameters in piglets with colitis. 


\section{MATERIAL AND METHODS}

\section{Animals and housing}

A total of 50 weaned piglets (Yorkshire $\times$ Songhei, 25 males and 25 females) of an average initial body weight of $4.50 \pm 0.68 \mathrm{~kg}$, weaned at $21 \pm 2$ day of age, were divided into five groups of ten each. Neonatal pigs were injected with ferrous gluconate at day 2 of life to prevent anaemia. $\mathrm{FeSO}_{4} \cdot 2 \mathrm{H}_{2} \mathrm{O}$ was supplemented in the diet before the start of study and for the study duration. Colitis was induced in piglets by giving $4 \%$ DSS in drinking water for the study duration, piglets without colitis received regular drinking water. After five days of acclimatization, the piglets were allocated to five dietary treatments of ten each in a randomized complete block design: 1 . control group: regular diet and water; 2 . DSS: regular diet $+4 \%$ DSS sodium in water; 3 . DSS $+\mathrm{Fe} 0.03 \%: 0.03 \% \mathrm{FeSO}_{4} \cdot 2 \mathrm{H}_{2} \mathrm{O}$ in diet $+4 \% \mathrm{DSS}$ in water; 4. DSS+Fe $0.2 \%: 0.2 \% \mathrm{FeSO}_{4}-2 \mathrm{H}_{2} \mathrm{O}$ in diet $+4 \% \mathrm{DSS}$ in water; 5 . DSS $+\mathrm{Fe} 0.1 \%$ : $1 \% \mathrm{FeSO}_{4} \cdot 2 \mathrm{H}_{2} \mathrm{O}$ in diet $+4 \%$ DSS in water. The piglets were housed individually in wire netted cage in a room with temperature maintained at $20 \pm 2^{\circ} \mathrm{C}$. The animals were fed the respective diets for 10 days, and diets were formulated to meet requirements according to NRC (1998).

\section{Sample collection and measurements}

On day 9 , to study the effect of iron on absorption and permeability of the intestinal mucosa in pigs, serum D-xylose and urinary Lac/Man ratio was measured. The test sugars were prepared as a test solution which contained a single dose, \%: lactulose 10, mannitol 5 and D-xylose 5. After an overnight fast and bladder emptying, the piglets were drenched $10 \mathrm{ml}$ of the test solution. After $2 \mathrm{~h}$ blood was collected, blood samples were centrifuged at $3600 \mathrm{rpm}$ at $4^{\circ} \mathrm{C}$ for $20 \mathrm{~min}$, and serum was separated and stored at $-80^{\circ} \mathrm{C}$ until analysed for serum concentration of D-xylose as markers of intestinal absorptive capacity. Simultaneously complete $6 \mathrm{~h}$ urine collection was made into a bottle containing preservative. The volume of each $6 \mathrm{~h}$ urine collection was recorded and a 10 -ml aliquot, preserved with thiomerasol, was used to analyse sugar concentration by high pressure liquid chromatography (HPLC) (Waters-600, USA) using a previously described method (Shippee et al., 1992). Lac/Man ratio as markers of mucosal permeability was analysed.

After the feeding trial, four pigs from each treatment group were slaughtered under general anaesthesia and then immediately eviscerated. The colon was collected and dissected out at $4^{\circ} \mathrm{C}$. The intestinal mucosa was isolated from these piglets as described by Heylings (1991). The mucosal homogenate was fractionated into $12.000 \mathrm{~g}$ supernatants as described earlier (Srigiridhar and Nair, 2000). 
Aliquots of supernatant were stored at $-80^{\circ} \mathrm{C}$ for further analysis. Protein in these supernatants was determined by the method of Lowry et al. (1951). Activities of SOD (EC1.15.1.1), CAT (EC1.11.1.6) and GPx (EC1.11.1.9) in colonic mucosa, were determined as described previously, in the mucosa as markers of oxidative stress (Srigiridhar and Nair, 1998). Plasma vitamin E was separated and quantified using a HPLC system (Waters 600, USA) on an RP-18 column with methanol as a solvent. A fluorescence spectrophotometer was used for the quantification of vitamin E. Lipid peroxidation in colon was evaluated by measuring MDA (Ohkawa et al., 1979), HP by the method of John and Steven (1978).

\section{Statistical analysis}

Differences among treatment groups were assessed by analysis of variance (ANOVA) using SPSS version 11.5 (SPSS, Chicago, IL, USA). Duncan's multiple range tests of significant differences among groups were determined. The values were considered significantly different if $\mathrm{P}$ values were $<0.05$, and expressed as mean and standard error of the mean (SEM).

\section{RESULTS}

We observed a markedly higher Lac/Man excretion ratio in piglets with DSS (Table 1). Furthermore, administration of $\mathrm{Fe} 0.03 \%$ in piglets did not increase the mean value for Lac/Man ratio when compared with piglets on DSS alone, but the Lac/Man ratio was significantly higher in Fe 0.2 and 1\% supplemented piglets than in other groups. On the other hand, the administration of DSS alone significantly decreased the serum level of D-xylose when compared with the piglets on control diet. However, addition of Fe $0.03 \%$ increased absorptive capacity compared with piglets on DSS alone. Furthermore, the level of D-xylose was significantly affected by Fe 0.2 and 1\% supplementation (Table 1).

Table 1. The urinary Lac/Man excretion ratio and the concentration of D-xylose in serum

\begin{tabular}{|c|c|c|c|c|c|}
\hline $\mathrm{mmol} / \mathrm{l}$ & Control & DSS & $\mathrm{DSS}+\mathrm{Fe} 0.03 \%$ & $\mathrm{DSS}+\mathrm{Fe} 0.2 \%$ & $\mathrm{DSS}+\mathrm{Fe} 1 \%$ \\
\hline Lac/Man & $0.097 \pm 0.006^{\mathrm{a}}$ & $0.189 \pm 0.010^{\mathrm{b}}$ & $0.150 \pm 0.009^{b}$ & $0.455 \pm 0.026^{c}$ & $0.773 \pm 0.043^{c}$ \\
\hline D-xylose & $4.20 \pm 0.23^{\mathrm{a}}$ & $3.61 \pm 0.03^{b}$ & $4.05 \pm 0.33^{\mathrm{ab}}$ & $2.22 \pm 0.11^{\mathrm{c}}$ & $2.03 \pm 0.17^{\mathrm{c}}$ \\
\hline
\end{tabular}


The activities of the scavenging enzymes SOD (Figure 1), GPx (Figure 2) and CAT (Figure 3) were significantly increased in the DSS group when compared with the control group. The addition of iron in the diet resulted in an inhibition of GPx and CAT activities, and their activities were significantly lower in Fe 0.2 and 1\% supplemented piglets than in piglets on DSS alone. In contrast piglets supplemented with various doses of iron, there was no
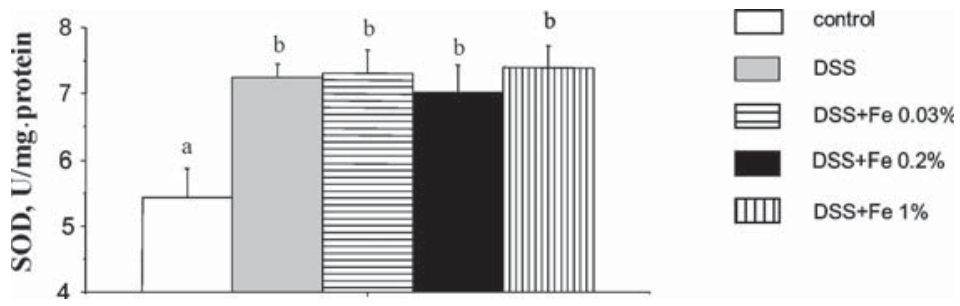

Figure 1. Effect of iron supplementation on superoxide dismutase (SOD) activity in colonic mucosa in piglets with induced colitis. Means with different letters are significantly different $(\mathrm{P}<0.05)$

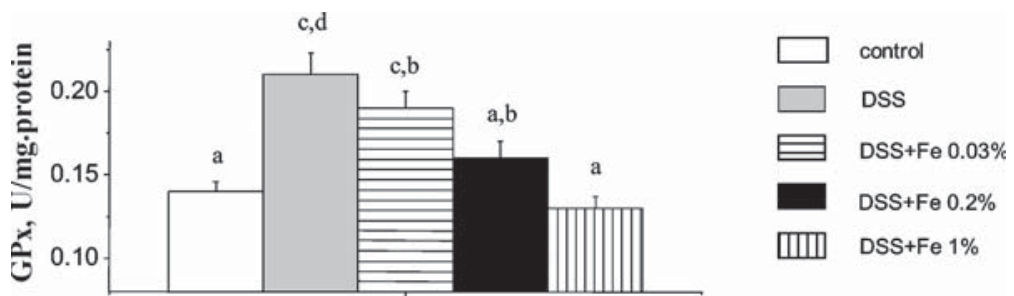

Figure 2. Effect of iron supplementation on glutathione peroxidase (GPx) activity in colonic mucosa in piglets with induced colitis. Means with different letters are significantly different $(\mathrm{P}<0.05)$
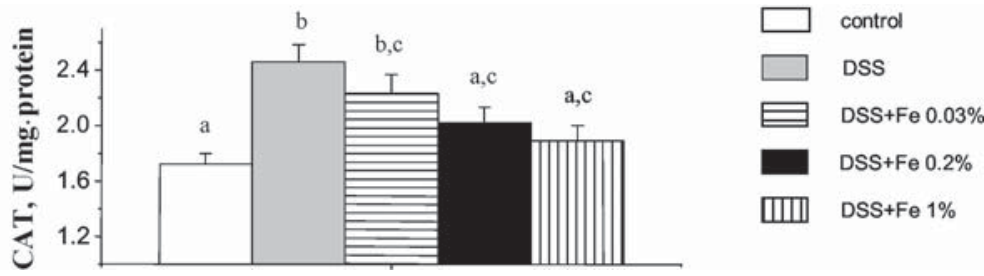

Figure 3. Effect of iron supplementation on catalase (CAT) activity in colonic mucosa in piglets with induced colitis. Means with different letters are significantly different $(\mathrm{P}<0.05)$

significant difference in activity of SOD between the DSS + Fe group and the DSS group. In addition, plasma levels of vitamin $\mathrm{E}$ in piglets supplemented with iron were significantly lower than in piglets on control diet in a dose-response manner (Figure 4). The production of MDA and HP as index of lipid peroxidation, was significantly increased by iron supplementation in colonic mucosa compared 
to the piglets on control diet (Table 2). Furthermore, various doses of iron supplementation significantly increased the accumulation of lipid peroxidation products, in a dose-response manner.
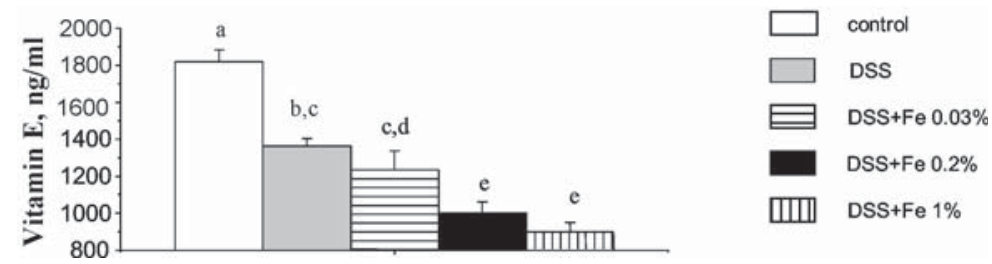

Figure 4. Effect of iron supplementation on vitamin E concentration in blood plasma in piglets with induced colitis. Means with different letters are significantly different $(\mathrm{P}<0.05)$

Table 2. Malondialdehyde and hydroperoxides in piglet colonic mucosa, $\mu \mathrm{M} / 100 \mathrm{~g}$ tissue

\begin{tabular}{lrcccc}
\hline & Control & DSS & DSS $+\mathrm{Fe} 0.03 \%$ & DSS $+\mathrm{Fe} 0.2 \%$ & DSS + Fe $1 \%$ \\
\hline MDA & $0.248 \pm 0.019^{\mathrm{a}}$ & $0.491 \pm 0.033^{\mathrm{b}}$ & $0.744 \pm 0.051^{\mathrm{c}}$ & $1.67 \pm 0.12^{\mathrm{d}}$ & $2.46 \pm 0.11^{\mathrm{e}}$ \\
HP & $8.35 \pm 0.53^{\mathrm{a}}$ & $17.53 \pm 1.55^{\mathrm{b}}$ & $28.93 \pm 1.60^{\mathrm{c}}$ & $80.87 \pm 4.31^{\mathrm{d}}$ & $112.76 \pm 3.90^{\mathrm{e}}$ \\
\hline
\end{tabular}

values expressed as mean \pm SEM, values in a row with non-identical superscripts are significantly different $(\mathrm{P}<0.05) ; \mathrm{n}=4$

\section{DISCUSSION}

Studies concerning iron deficiency in piglets have been focused mainly on different methods of iron supplementation, and little attention has been paid to the impairment of excessive iron on intestine of piglets with IBD. Iron deficiency is a common phenomenon in piglets with IBD. However, there is little real data available on how the oral iron influences the intestinal permeability and oxidative stress in weaned piglets with IBD.

Many kinds of ferrous compounds can reduce gastrointestinal side effects by oral supplementation. Rimon et al. (2005) reported oral $50 \mathrm{mg}$ of ferrous gluconate or $150 \mathrm{mg}$ of ferrous calcium citrate result in abdominal discomfort, nausea, vomiting, changes in bowel movements, and black stools in human, and significantly more common at higher iron doses. Thus the main reason for gastrointestinal side effects induced by oral ferrous compounds was excess $\mathrm{Fe}^{2+}$. During intestinal inflammation, circulating neutrophils of the inflamed mucosa amplified the inflammation by releasing $\mathrm{H}_{2} \mathrm{O}_{2}$ and $\mathrm{O}_{2}^{-}$. Via Haber-Weiss reaction (Imlay et al., 1988), $\mathrm{Fe}^{2+}$ reacts with these free radicals to produce a number of $\mathrm{OH} \cdot$ in the intestinal mucosa with IBD. Our data show that piglets supplemented with iron resulted in a significant decrease in plasma vitamin $\mathrm{E}$ in line with Carrier 
et al. (2001). The main reason for the decrease in mucosal vitamin $\mathrm{E}$ would be that hydroxyl radical increases in colonic mucosa, and vitamin E mainly scavenges hydroxyl and peroxyl radicals (Huang et al., 2002), furthermore lowers feed intake of the piglets with IBD. Schweigert et al. (2000) reported that a lower concentration of vitamin $\mathrm{E}$ in iron-treated piglets might be a consequence of iron-associated mechanism that lead to a disappearance of antioxidative vitamins from plasma. IBD significantly increased the activities of mucosal antioxidant enzyme in DSS group compared with the control group. This may be due to feed back activation of antioxidant enzyme synthesis. However, the high dose of iron supplementation, led to a decrease in CAT and GPx activities, but not in SOD activity in Fe 0.2 and 1\% groups. Connye and Kuratko (1999) reported that supplemental iron may increase MnSOD due to increased oxidative stress but did not compromise the ability of inflammatory mediators to further increase the activity of this enzyme. It is known that superoxide anion can inactivate GPx and CAT, $\mathrm{H}_{2} \mathrm{O}_{2}$ can inactivate SOD. It can be hypothesized that the reasons for the decrease in CAT and GPx activities, but not SOD, in $\mathrm{Fe} 0.2$ and $1 \%$ groups would be that increased levels of $\mathrm{O}_{2}^{-}$, inactivate CAT and GPx. The ferrous reacted with $\mathrm{H}_{2} \mathrm{O}_{2}$ to produce $\mathrm{OH}$, and reduced the amount of $\mathrm{H}_{2} \mathrm{O}_{2}$.

Increased amount of ROS would lead to an exhaustion of defence. Thus, the enhancement in the concentration of lipid peroxidation products in piglets with oxidative stress was not surprising. In the present study, the addition of iron in the diet resulted in an increase of MDA and HP in colonic mucosa. These data suggest that iron played a significant role in the tissue with induced damage, and piglets fed high iron levels were more susceptible to peroxidative damage under the challenge of IBD. Kadis et al. (1984) and Weinberg (1987) reported that piglets fed with excessive iron would increase susceptibility to bacteria and result in diarrhoea and infection. These results show that iron inflicted oxidative damage on the intestinal mucosa.

High ROS further enhanced inflammation by improving mucosal permeability (Weinberg, 1987). Intestinal mucosal disease is often associated with altered permeability, usually with increased permeability for larger molecules. We found that intestinal permeability expressed as urinary Lac/Man excretion was significantly raised in piglets supplied with high iron levels. Shippee et al. (1992), using a combination of lactulose and mannitol comparing 80 cirrhotics with 28 normals, found the mean Lac/Man permeability ratio to be higher than for control subjects. Maintenance of the barrier depends on the integrity of cellular plasma membranes and tight junctions, as well as the elaboration of endothelial and epithelial secretory products. ROS destroy intestinal barrier integrity by lipid peroxidation (Sreedhar et al., 2004). Travis and Menzies (1992) suggested that the increase in intestinal permeability indicates damage of mucosal barrier function. Focal denudation of the barrier results in permeation of potential threat such as 
antigens, proteases, bacteria and endotoxin for inflammatory cells. We found that iron supplementation led to a decrease in serum D-xylose. Kuratko (1998) reported that a high level of dietary iron caused intestinal barrier dysfunction, consequently decreased intestinal absorptive capacity in vivo. The clinically significant malabsorption due to IBD may be promoted by deleterious oxidants released from inflammatory cells that accumulate within the intestinal interstitium during active disease (Grisham and Granger, 1988). Hence, the intestinal brush border membrane is of critical importance for the normal absorption of dietary nutrients.

\section{CONCLUSIONS}

Excess iron supplementation caused injury on intestinal mucosa in piglets with colitis, these might be involved in the antioxidant defence system weaken in condition which the oxidative stress was stronger and sustained. Thus, lower iron supplementation in diarrhoetic and iron-deficient piglets might be beneficial.

\section{ACKNOWLEDGEMENTS}

The authors are grateful to $\mathrm{Li} \mathrm{Na}$ and Xiaodong Zhao for assistance with the experiments.

\section{REFERENCES}

Bowlus C.L., 2003. The role in T cell development and autoimmunity. Autoimmunol. Rev. 2, 73-78

Brennan L.A., Morris G.M., Wasson G.R., Hannigan B.M., Barnett Y.A., 2000. The effect of vitamin $\mathrm{C}$ or vitamin $\mathrm{E}$ supplementation on basel and $\mathrm{H}_{2} \mathrm{O}_{2}$ induced DNA damage in human lymphocytes. Brit. J. Nutr. 84, 195-202

Carrier J., Aghdassi E., Platt I., Cullen J., Allard J.P., 2001. Effect of oral iron supplementation on oxidative stress and colonic inflammation in rats with induced colitis. Aliment. Pharmacol. Therapeut. 15, 1989-1999

Connye N., Kuratko C., 1999. Iron increases manganese superoxide dismutase activity in intestinal epithelial cells. Toxicol. Lett. 104, 151-158

Daykin M M., Griffiths A.J., Towlerton R.G., 1982. Evaluation of the parenteral iron requirement of early weaned pigs. Vet. Res. 110, 535-537

Erichsen K., Hausken T., Berge R., Ulvik R., Berstad A., 1998. Effect of oral iron therapy on antioxidant defence in active Crohn's disease. Digestion 59, 1287

Grisham M.B., Granger D.N., 1988. Neutrophil-mediated mucosal injury: role of reactive oxygen metabolites. Digest. Dis. Sci. 33, 6-15

Heylings J.R., 1991. Gastrointestinal absorption of paraquat in the isolated mucosa of the rat. Toxicol. Appl. Pharmacol. 107, 482-493 
Huang R.F., Huang S.M., Lin B.S., Hung C.Y., Lu H.T., 2002. N-acetylcysteine, vitamin C and vitamin $\mathrm{E}$ diminish homocysteine thiolactone-induced apoptosis in human promyeloid HL-60 cells. J. Nutr. 132, 2151-2156

Imlay J.A., Chin S.M., Linn S., 1988. Toxic damage through the Fenton reaction in vivo and in vitro. Science 240, 640-642

John A.B., Steven D.A., 1978. Microsample lipid peroxidation. In: S. Fleischer, L. Packer (Editors). Methods in Enzymology. 52. Academic Press, New York, pp. 302-310

Kadis S., Vdeze F.A., Polanco J., 1984. Relationship of iron administration to susceptibility of newborn pigs to enterotoxic colibacillosis. Amer. J. Vet. Res. 45, 255-259

Kuratko C., 1998. Fatty acids and iron modulate manganese content and c manganese superoxide dismutase activity in colonic mucosa during early stages of carcinogenesis. Food Chem. Toxicol. $36,819-824$

Lowry O.H., Rosebrough N.J., Forr A.L., Randall R.J., 1951. Protein measurement with the Folin phenol reagent. J. Biol. Chem. 193, 265-275

Manashi B.D., Mark M.B.S., Case W.B.S., 2000. The acute and chronic stress-induced oxidative gastrointestinal injury in rats, and the protective ability of a novel grape seed proanthocyanidin extract. Nutr. Res. 19, 1189-1199

NRC, 1998. Nutrition Requirements of Swine. 10th revised Edition. National Academy Press. Washington, DC, pp. 110-116

Ohkawa H., Ohishi N., Yagi K., 1979. Assay for lipid peroxidation in animal tissues by thiobarbituric acid reaction. Anal. Biochem. 95, 351-358

Rimon E., Kagansky N., Kagansky M., Mechnick L., Mashiah T., Namir M., Levy S., 2005. Are we giving too much iron? Low-dose iron therapy is effective in octogenarians. Amer. J. Med. 118, $1142-1147$

Schweigert F.J., Gürtler H., Baumane A., Wahren M., Leo M., 2000. Effect of iron supplementation on plasma levels of vitamins A, E and C in piglets. Livest. Prod. Sci. 63, 297-302

Shippee R.L., Johnson A.A., Cioffi W.G., 1992. Simultaneous determination of lactulose and mannitol in urine of burn patients by gas/liquid chromatography. Clin. Chem. 38, 343-345

Sreedhar B., Subramaniyan R., Nair K.M., 2004. A protective role for zinc on intestinal peroxidative damage during oral iron repletion. Biochem. Biophys. Res. Commun. 318, 992-997

Srigiridhar K., Nair K.M., 1998. Iron-deficient intestine is more susceptible to peroxidative damage during iron supplementation in rats. Free Radical Biol. Med. 25, 660-665

Srigiridhar K., Nair K.M., 2000. Supplementation with alpha-tocopherol or a combination of alphatocopherol and ascorbic acid protects the gastrointestinal tract of iron-deficient rats against ironinduced oxidative damage during iron repletion. Brit. J. Nutr. 84, 165-173

Srigiridhar K., Nair K.M., Subramanian R., Singotamu L., 2001. Oral repletion of iron induces free radical mediated alterations in the gastrointestinal tract of rat. Mol. Cell. Biochem. 219, 91-98

Svoboda M., Drabek J., Krejci J., Rehakova Z., Faldynal M., 2004. Impairment of the peripheral lymphoid compartment in iron-deficient piglets. J. Vet. Med. B 51, 231-237

Travis S., Menzies I., 1992. Intestinal permeability: functional assessment and significance. Clin. Sci. Colch. 82, 471-488

Urban T., Hurbain I., Urban M., Clement A., Housse B., 1995. Oxidants and antioxidants. Biological effects and therapeutic perspectives. Ann. Chir. 49, 427-434

Weinberg E.D., 1987. Iron and infection. Microbiol. Res. 42, 45-66 